\title{
A Subcutaneous Insulin Pharmacokinetic Model for Insulin Detemir
}

\author{
Ben van Noorden, Jennifer L. Knopp, J. Geoffrey Chase \\ University of Canterbury, Dept of Mechanical Engineering, Centre for Bio-Engineering, Christchurch, \\ New Zealand;
}

Corresponding Author Email: Geoff.chase@canterbury.ac.nz

\begin{abstract}
: 327 words
Background and Objective: Type 2 diabetes (T2D) is rapidly increasing in incidence and has significant social and economic costs. Given the increasing cost of complications, even relatively short delays in the onset of T2D can significantly reduce long-term complications and costs. Equally, recent studies have shown the onset of T2D can be delayed by use of long-acting insulin, despite the risk and concomitant low adherence. Thus, there is a strong potential motivation to develop models of longacting insulin analogues to enable safe, effective use in model-based dosing systems. In particular, there are no current models of long-acting insulin Detemir and its unique action for model-based control. The objective of this work is to develop a first model of insulin Detemir and its unique action, and validate it against existing data in the literature.
\end{abstract}

Methods: This study develops a detailed compartment model for insulin Detemir. Model specific parameters are identified using data from a range of published clinical studies on the pharmacokinetic of insulin Detemir. Model validity and robustness are assessed by identifying the model for each study and using average identified parameters over several dose sizes and study cohorts. Comparisons to peak concentration, time of peak concentration and overall error versus measured plasma concentrations are used to assess model accuracy and validity.

Results: Almost all studies and cohorts fit literature data to within one standard deviation of error, even when using averaged identified model parameters. However, there appears to be a noticeable dose dependent dynamic not included in this first model, nor reported in the literature studies.

Conclusions: A first model of insulin Detemir including its unique albumin binding kinetics is dervied and provisionally validated against clinical pharmacokinetic data. The pharmacokinetic curves are suitable for model-based control and general enough for use. While there are limitations in the studies used for validation that prevent a more complete understanding, the results provide an effective first model and justify the design and implementation of further, more precise human trials.

Keywords: Insulin Detemir; Physiological Model; Compartment Model; Diabetes; Pharmacokinetics

Conflict of Interest: The authors state they have no conflict of interest or financial interest in the study or the results. 


\subsection{Introduction:}

Type 2 Diabetes (T2D) is characterised by insulin resistance followed by reduced and eventually nonexistent endogenous insulin production, all of which lead to increasing and persistent hyperglycemia. Worldwide 400 million people live with T2D and it is estimated almost 200 million more people have undiagnosed diabetes, with significant increases of $30-50 \%$ predicted by 2030 [1-4]. Early detection and treatment reduces morbidity and mortality, improves quality of life, and thus reduces the social and economic healthcare burden [5].

Normal endogenous insulin secretion is bi-phasic, with a first phase impulse response to a meal added to a relatively constant basal secretion rate. Insulin analogues have been developed to replicate these two behaviours, with fast acting analogues used to replicate the impulse response, and slow acting analogues used to replicate basal secretion. Insulin Detemir ${ }^{\text {TM }}$ (Nov Nordisk, Denmark) is one such slow acting basal insulin analogue [6]. However, while insulin therapy is commonly used in individuals with very high insulin resistance and/or failed insulin secretion, it is a last resort therapy in T2D for safety reasons [7-9].

Insulin Detemir is subcutaneously injected as a hexameric unit, which then dissociates into monomers and dimers in the subcutaneous interstitium [10]. Insulin Detemir differs from endogenous human insulin in that a C14 fatty acid chain has been attached to the amino acid B29, and the amino acid threonine at B30 is omitted [11], which, different to other long acting analogues, allows it to bind to albumin in both interstitial fluid and blood plasma. This binding means only $2-4 \%[10,12]$ of insulin Detemir is available for receptor binding to mediate glucose uptake at any time. Its action is further prolonged due to strong self-association in the hexameric form [10].

However, despite the rise of computer and model based glycemic control using insulin pumps [13-15], there are no current models of insulin Detemir and its unique action for use in model-based control methods for those type 1 and type 2 diabetic individuals using injected insulin (e.g. [16]). In particular, prior models of long acting insulin analogues do not include this albumin binding aspect [17-19], which makes the model unique in its own right. 
Equally, a validated model can be used to guide insulin dosing in model-based protocols for those using multiple daily injection to control glucose levels. A better understanding of the appearance profile would enable safe, more accurate control versus using current models for other analogues. In particular, while the model parameters can be modified in other insulin analogue models to provide an estimated appearance profile, it is not necessarily accurate, and they cannot model or account for both bound and unbound Detemir masses, all of which can lead to error and increased risk.

This study aims to create a physiologically relevant pharmacokinetic model for the appearance of insulin Detemir in plasma and interstitium from an injection depot site. Data from pharmacokinetic studies in the literature are used for initial model validation, including a type 1 diabetic cohort and a type 2 diabetic cohort, where data on these specialised cohorts is often lacking. This pharmacokinetic model could prove useful in model-based glycemic control using multiple daily injection in lieu of insulin pumps to treat diabetes. More immediately, a validated model would also justify clinical studies on specific type 1 and type 2 diabetes cohorts to optimise model parameters, as well as understand the variability of appearance, which is not possible with current data sets in the literature. 


\subsection{Methods:}

\subsection{Model:}

A pharmacokinetic model of insulin Detemir in the subcutaneous (SC) fluid and plasma serum is presented pictorially in Figure 1. It contains all relevant compartments and bound/unbound states. In the local injection depot, three compartments describe hexameric Detemir $\left(I_{D H}, m U\right)$, and both unbound $\left(Q_{D F, L o c a l}, m U\right)$ and bound $\left(Q_{D B, l o c a l}, m U\right)$ monomer/dimer Detemir in the local depot. Table 1 defines the key model variables from Figure 1 and subsequent development.

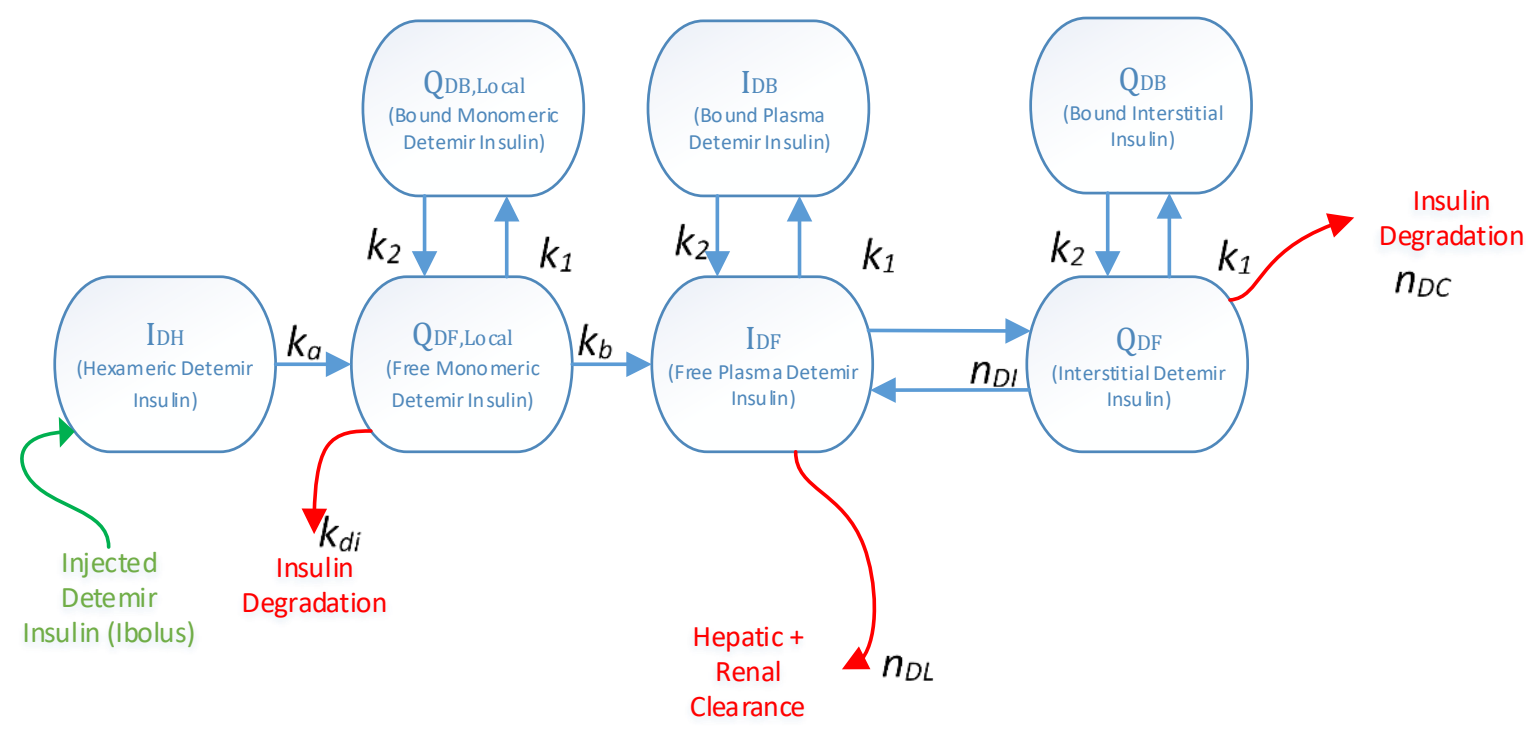

Figure 1: Schematic of compartment model for the pharmacokinetics of Insulin Detemir

Table 1: Summary and definition of parameters used in the model

\begin{tabular}{|c|c|c|c|c|}
\hline Parameter & Value & Units & Source & Definition \\
\hline$I_{\text {Bolus }}$ & $0.15-0.75$ & $\mathrm{mU} \cdot \mathrm{kg}^{-1}$ & [20-22] & Subcutaneous insulin Detemir injected \\
\hline$k_{a}$ & 0.0078 & $\min ^{-1}$ & [23] & $\begin{array}{l}\text { Rate constant for the hexameric dissociation } \\
\text { to dimers/monomers }\end{array}$ \\
\hline$k_{b}$ & & $\min ^{-1}$ & identified & $\begin{array}{l}\text { Rate constant for the diffusion of the unbound } \\
\text { insulin from the local interstitium into the blood } \\
\text { plasma }\end{array}$ \\
\hline$k_{1}$ & 0.96 & $\min ^{-1}$ & {$[10,12]$} & $\begin{array}{l}\text { Rate constant defining the binding of the } \\
\text { insulin to albumin }\end{array}$ \\
\hline$k_{2}$ & 0.04 & $\min ^{-1}$ & {$[10,12]$} & $\begin{array}{l}\text { Rate constant defining the unbinding of the } \\
\text { insulin to albumin }\end{array}$ \\
\hline$k_{d i}$ & & $\min ^{-1}$ & identified & $\begin{array}{l}\text { Degradation rate constant of unbound insulin } \\
\text { in the local depot }\end{array}$ \\
\hline$V_{I}$ & 4.0 & $\mathrm{~L}$ & [24] & Plasma distribution volume \\
\hline$n_{D L}$ & & $\min ^{-1}$ & identified & Hepatic and renal insulin clearance rates \\
\hline$n_{D I}$ & 0.06 & $\min ^{-1}$ & [24] & $\begin{array}{l}\text { Trans-endothelial diffusion rate between the } \\
\text { blood plasma and the interstitial fluid }\end{array}$ \\
\hline$n_{D C}$ & 0.032 & $\min ^{-1}$ & [24] & $\begin{array}{l}\text { Rate of degradation of the Detemir in the } \\
\text { interstitial fluid }\end{array}$ \\
\hline
\end{tabular}


These compartments are defined:

$$
\begin{gathered}
\dot{I}_{D H}=-k_{a} I_{D H}+I_{B o l u s} \delta(t) \\
\dot{Q}_{D F, \text { Local }}=k_{a} I_{D H}-\left(k_{b}+k_{d i}\right) Q_{D F, \text { local }}-\left(k_{1} Q_{D F, \text { local }}-k_{2, I F} Q_{D B, \text { local }}\right) \\
\dot{Q}_{D B, \text { Local }}=k_{1} Q_{D F, \text { local }}-k_{2} Q_{D B, \text { local }}
\end{gathered}
$$

Where $I_{\text {Bolus }}$ is the amount of subcutaneously injected insulin Detemir, $\delta$ is the Dirac delta function, $k_{a}$ is the rate constant for the hexameric dissociation to dimers/monomers, $k_{b}$ is the rate constant for the diffusion of the unbound insulin from the local interstitium into the blood plasma, $k_{d i}$ is the degradation of the unbound insulin in the local depot, $k_{1}$ is the rate constant defining the binding of the insulin to albumin, and $k_{2}$ is the rate constant defining the unbinding of the insulin to albumin. As evidence suggests dimers and monomers diffuse between plasma and interstitium at similar rates [25], and both dimers and monomers of this insulin analogue bind to albumin, these masses are combined.

Insulin Detemir diffuses from the local depot into blood plasma, where it circulates and is free to equilibrate with the whole-body interstitial fluid compartment. Specifically, in Figure 1, $I_{D F}(m U / L)$ is the 'free' unbound insulin Detemir in the blood plasma, $I_{D B}(\mathrm{mU} / \mathrm{L})$ is the bound insulin Detemir in the plasma, $Q_{D F}(m U / L)$ is the unbound insulin Detemir in the interstitial fluid and $Q_{D B}(m U / L)$ is the bound insulin in the interstitial fluid. These compartment models are defined:

$$
\begin{gathered}
\dot{I}_{D F}=\frac{k_{b}}{V_{I}} Q_{D F, l}-\left(n_{D L}\right) I_{D F}-n_{D I}\left(I_{D F}-Q_{D F}\right)-\left(k_{1} I_{D F}-k_{2} I_{D B}\right) \\
\dot{I}_{D B}=k_{1} I_{D F}-k_{2} I_{D B} \\
\dot{Q}_{D F}=-n_{D C} Q_{D F}+n_{D I}\left(I_{D F}-Q_{D F}\right)-\left(k_{1} Q_{D F}-k_{2} Q_{D B}\right) \\
\dot{Q}_{D B}=k_{1} Q_{D F}-k_{2} Q_{D B}
\end{gathered}
$$

Where, in Equations (4)-(7) and in Table 1, $V_{I}$ is the volume of distribution ( $L$ ) of the insulin Detemir, which is assumed similar to that of human insulin. The parameter $n_{D L}$ determines the hepatic and renal insulin clearances, $n_{D I}$ the trans-endothelial diffusion rate between the blood plasma and the interstitial fluid, $k_{1}$ the rate of binding of Detemir to albumin, $k_{2}$ the rate of unbinding of Detemir from albumin, and 
$n_{D C}$ the rate of degradation of the Detemir in the interstitial fluid. Constants $k_{1}$ and $k_{2}$ govern binding and unbinding to albumin, and are assumed constant across the depot, plasma, and interstitial fluid, and are valued so at steady state $96 \%$ of insulin is bound to plasma albumin per literature reports $[10,12,26-28]$. Binding is a non-saturated process as albumin is plentiful relative to Detemir dose [10].

Values in Table 1 are defined from other models of human insulin pharmacokinetics in the literature for common kinetics. The parameter values identified from data in this study are specific to Detemir, and include $k_{b}, k_{d i}$, and $n_{D L}$, which are not previously reported elsewhere and are thus assumed to describe the dynamics most specific to insulin Detemir. These identified parameters were also selected based on model identifiability [29], and for their sensitivity in controlling the model dynamics in terms of peak concentration and clearance rates of insulin Detemir, as measured in plasma clinically. In particular, the clearance $n_{D C}$ is known to trade off with $n_{D L}$, so $n_{D C}$ is set to a human insulin value, and only $n_{D L}$ is uniquely identified. The input value of the bolus, $I_{B o l u s}$, is the experimental input controlled and recorded in each study.

\subsection{Data:}

A literature search of PubMed and Google was carried out to find experimental studies of insulin Detemir pharmacokinetics, using combinations of the search terms "Detemir", "Insulin", "NN304" and "pharmacokinetics". Five studies were found [20-22,30,31] which measured and reported serum insulin Detemir, where serum is defined as the total amount of bound and unbound insulin Detemir in blood plasma. The studies used a wide range of doses from $0.15-0.75 \mathrm{U} / \mathrm{kg}$, with equally varied cohorts and protocols, and are summarised in Table 2.

Briefly, the study by Jhee et al compared Detemir pharmacodynamics in American Caucasians and Americans of Japanese heritage [22]. Brunner et al examined the pharmacokinetics of Detemir at different doses, and compared this to NPH insulin [20]. Heinemann et al examined the time action profile of insulin Detemir at three different doses [30], while Danne et al examined differences in the pharmacokinetic profiles between adults and adolescents with T1D [21]. Morrow et al examined the additive effect of Detemir and liraglutide in subjects with T2D [31]. Where units differed between studies and models, the conversion factor of $1 \mu \mathrm{U} / \mathrm{L}=6.0 \mathrm{pmol} / \mathrm{L}$ was used for plasma insulin [32]. There is 
thus a range of doses, subjects ( $\mathrm{N}=100$ total), and clinical contexts, enabling identification of a general model across all of them that is not specific to a particular cohort or context.

Table 2: Studies reporting PK profiles and data for insulin Detemir. All values are mean \pm SD where relevant and reported.

\begin{tabular}{|c|c|c|c|c|c|c|c|c|c|}
\hline Paper & $\begin{array}{l}\# \\
\text { Subjects }\end{array}$ & $\begin{array}{l}\text { Age } \\
\text { (years) }\end{array}$ & $\begin{array}{l}\text { BMI } \\
\left(\mathrm{kg} / \mathrm{m}^{2}\right)\end{array}$ & $\begin{array}{l}\text { Weight } \\
\text { (kg) }\end{array}$ & $\begin{array}{l}\text { Injection } \\
\text { Location }\end{array}$ & $\begin{array}{l}\text { Bolus } \\
\text { Amount } \\
\text { (U/kg) }\end{array}$ & $\begin{array}{l}C_{\max } \\
\text { (pmol/L) }\end{array}$ & $\begin{array}{l}t_{\max } \\
\text { (hrs) }\end{array}$ & $\begin{array}{l}\text { AUC } \\
(\mathrm{pmol} \cdot \mathrm{hrs} / \mathrm{L})\end{array}$ \\
\hline $\begin{array}{l}\text { Jhee et al. } 2004 \\
\text { [22] } \\
\text { Healthy Caucasian }\end{array}$ & 16 & $33.5 \pm 9.9$ & $24.9 \pm 2.6$ & $78.8 \pm 10.9$ & Thigh SC & 0.19 & $1.82 \pm 0.5^{x}$ & $5.38 \pm 2.01$ & $0.99 \pm 0.15^{x x}$ \\
\hline \multirow{2}{*}{$\begin{array}{l}\text { (Jhee et al. 2004) } \\
\text { [22] } \\
\text { Healthy with } \\
\text { Japanese heritage }\end{array}$} & \multirow[b]{2}{*}{20} & \multirow[b]{2}{*}{$22.7 \pm 4.0$} & \multirow[b]{2}{*}{$21.9 \pm 2.8$} & \multirow[b]{2}{*}{$60.1 \pm 8.3$} & \multirow{2}{*}{ Thigh SC } & 0.19 & $1.74 \pm 0.49 x$ & $4.53 \pm 1.97$ & $0.97 \pm 0.15^{x x}$ \\
\hline & & & & & & 0.38 & $3.18 \pm 0.89 x$ & $6.03 \pm 2.62$ & $2.11 \pm 0.32^{x x}$ \\
\hline $\begin{array}{l}\text { Brunner et al. } 2000 \\
{[20]-\text { Healthy }} \\
\text { males }\end{array}$ & 10 & $26.2 \pm 3.5$ & $23.0 \pm 1.5$ & - & $\begin{array}{l}\text { Para- } \\
\text { umbilical } \\
\text { skinfold }\end{array}$ & 0.6 & $1.81 \pm 0.48$ & $4.5 \pm 2.07$ & $0.93 \pm 0.14$ \\
\hline \multirow{3}{*}{$\begin{array}{l}\text { Heinemann et al. } \\
1999 \text { [30] - Healthy } \\
\text { males }\end{array}$} & \multirow{3}{*}{11} & \multirow{3}{*}{$27 \pm 2$} & \multirow{3}{*}{$24.1 \pm 1.7$} & \multirow{3}{*}{-} & \multirow{3}{*}{$\begin{array}{l}\text { Para- } \\
\text { umbilical } \\
\text { skinfold }\end{array}$} & 0.15 & $0.61 \pm 0.14$ & $4.45 \pm 1.4$ & $0.34 \pm 0.04$ \\
\hline & & & & & & 0.3 & $1.05 \pm 0.28$ & $5.42 \pm 1.6$ & $0.67 \pm 0.08$ \\
\hline & & & & & & 0.6 & $2.03 \pm 0.46$ & $5.37 \pm 1.2$ & $1.23 \pm 0.21$ \\
\hline $\begin{array}{l}\text { Danne et al. } 2003 \\
\text { [21] - T1D adults }\end{array}$ & 11 & $22.8 \pm 6.4$ & $23.4 \pm 1.9$ & $76.5 \pm 6.7$ & Thigh SC & 0.5 & $4.64 \pm 2.30$ & $8.05 \pm 3.43$ & $3.38 \pm 1.42$ \\
\hline
\end{tabular}

x SD not given in paper so value of $28 \%$ is used based on Brunner and Heinemann values

${ }^{x \times}$ SD not given in paper so value of $15 \%$ is used based on Brunner and Heinemann values

* SD not given in paper so value of $49 \%$ is used based on Danne value

** SD not given in paper so value of $43 \%$ is used based on Danne value

*** SD not given in paper so value of $42 \%$ is used based on Danne value

Reported values of peak concentration and its time of occurrence $\left(C_{\max }, t_{\max }\right)$, as well as the AUC of measured serum insulin Detemir are also summarised in Table 2, and will be compared to identified model values. Where estimations of variance were missing, standard deviations (SDs) have been computed based on SD's as a \% of the mean value from the study (i.e. Standard Error), or studies most similar to the current study. This latter approximation was made for data from Jhee et al and Morrow et al in the absence of any better data to assess variability, where such an assumption assumes only that insulin assays and sampling procedures have similar variability across studies, which is not unreasonable.

\subsection{Model Identification:}

Values for $k_{d i}, k_{b}$, and $n_{D L}$ are identified using data from the studies in Table 2 using a Gauss Newton parameter identification algorithm. The numerical Jacobian was calculated using a parameter 
perturbation of $\pm 1 \%$ of the parameter value. To stabilise convergence, the new parameter estimate was defined as $10 \%$ of the new Gauss Newton estimate added to $90 \%$ of the previous parameter values, limiting step size for convergence. Model errors were plotted to ensure convergence to steady state was obtained, where convergence was measured as a change in least squares error less than $1.0 \mathrm{e}-4$ between iterations. Least squares errors were calculated as the difference between literature reported serum Detemir insulin and the modelled sum of plasma free and bound Detemir insulin $\left(I_{D F}+I_{D B}\right)$ to match the published reports.

The model is theoretically and practically identifiable [33]. However, there is also some trade off of the dynamic effect each variable has on the final model solution, even if the identified solution is optimal for the data used in the identification process. Specifically, the value of $k_{d i}$ mainly affects $C_{\max }$, while $k_{b}$ affects primarily $t_{\max }$, and $n_{D L}$ primarily affects the clearance/disappearance decay slope, but in all cases it is the net overall effect that yields the final curve. The identification approach provides a global optimum, and the generalisability of the results to reported experimental data will assess whether the identified values are robust.

\subsection{Comparison to literature reported PK outcomes:}

Area under the curve $(A \cup C)$, maximum serum concentration $\left(C_{\max }\right)$ and time of maximum concentration $\left(t_{\max }\right)$ for the identified model results are compared to those from the published studies in Table 2. After values are identified for each individual data set and compared to the reported PK values for that data set in Table 2, the mean identified parameter values across an entire study are used to assess model robustness in a further comparison to the reported data, as well as assessing the robustness of the model dynamics and parameters as identified. Finally, the identified parameters for the two best performing data sets are averaged and simulated to assess inter-study robustness and variability of the model.

The identified model was assessed as accurate and robust if the model using identified average parameter values was within 1 standard deviation of the measured data set value, thus accounting for measurement error with a very conservative metric. 


\subsection{Results:}

\subsection{Identification and Identified Model Fit:}

Model parameter values identified from literature data for each data set across all studies are shown in Table 3 and corresponding model fits in Figure 2. The model did not converge for the data from the Morrow et al studies [31], potentially due to the very specific diabetes cohort used yielding very different curves from the model dynamics, or equally due to measurement or other study errors. The results for Morrow et al are thus not presented. The data from Brunner et al and Heinemann et al had considerably lower serum insulin Detemir concentrations than Jhee et al at higher initial bolus amounts, showing significant variability in the reports, as well, which yield different pharmacokinetic parameter values.

Table 3: Model parameters fit from literature

\begin{tabular}{|l|l|l|l|l|}
\hline Paper & $\begin{array}{l}\text { Bolus } \\
\text { Amount }\end{array}$ & $\begin{array}{c}\boldsymbol{k}_{\boldsymbol{d i}} \\
\left(\mathbf{m i n}^{-\mathbf{1}}\right)\end{array}$ & $\begin{array}{c}\boldsymbol{k}_{\boldsymbol{b}} \\
\left(\mathbf{m i n}^{-\mathbf{1}}\right)\end{array}$ & $\begin{array}{c}\boldsymbol{n}_{\boldsymbol{D L}} \\
\left(\mathbf{m i n}^{-\mathbf{1}}\right)\end{array}$ \\
\hline \multirow{3}{*}{ Chee et al. 2004 [22] } & 0.19 & 0.0530 & 0.0155 & 0.3344 \\
\cline { 2 - 5 } & 0.38 & 0.0720 & 0.0191 & 0.2746 \\
\cline { 2 - 5 } & 0.75 & 0.0754 & 0.0080 & 0.1071 \\
\hline \multirow{3}{*}{ Japee et al. 2004 [22] } & 0.19 & 0.0378 & 0.0388 & 0.6270 \\
\cline { 2 - 5 } & 0.38 & 0.0456 & 0.0163 & 0.2767 \\
\cline { 2 - 5 } & 0.75 & 0.0730 & 0.0106 & 0.1069 \\
\hline \multirow{3}{*}{ Hrunner et al. 2000 [20] } & 0.3 & 0.0605 & 0.0506 & 2.1227 \\
\cline { 2 - 5 } & 0.6 & 0.0635 & 0.0258 & 1.4148 \\
\hline Heinemann et al. 1999 [30] & 0.15 & 0.0705 & 0.0194 & 0.7222 \\
\cline { 2 - 5 } & 0.3 & 0.0803 & 0.0085 & 0.3195 \\
\cline { 2 - 5 } & 0.6 & 0.0920 & 0.0082 & 0.2884 \\
\hline Danne et al. 2003[21] & 0.5 & 0.0444 & 0.0072 & 0.1344 \\
\hline Morrow et al. 2011[31] & 0.5 & \multicolumn{3}{|c}{ Did not converge } \\
\hline
\end{tabular}

Averaged parameter values across each study are shown in Table 4. PK parameters for human insulin from Lotz et al [24], Wong [23] and Shimoda et al [34], are presented for comparison. The value of $n_{D L}$ is close to the human insulin literature value in general and especially for the Jhee et al and the Heinemann et al cohorts. The Brunner et al averaged value of $n_{D L}$ is not physiologically realistic as it is extremely high [24]. The other results and comparisons show broadly similar values although the identified value for $k_{b}$ for Danne et al is significantly lower than the others and the added comparator studies. Overall, there is variability both between studies and within studies, potentially reflective of different cohorts and/or dose specific dynamics, or due to assumptions made in translating the data for comparison. 
Table 4: The averaged parameters from the values found in Table 3 where the latter three results are shown for comparison to common values identified in prior studies for added comparison. The Jhee et al data are averaged across the Caucasian and Japanese cohorts in Table 3.

\begin{tabular}{|l|l|l|l|}
\hline Paper & \multicolumn{1}{|c|}{$\boldsymbol{k}_{\boldsymbol{d i}}$} & \multicolumn{1}{c|}{$\boldsymbol{k}_{\boldsymbol{b}}$} & \multicolumn{1}{c|}{$\boldsymbol{n}_{\boldsymbol{D L}}$} \\
\hline Jhee et al. 2004 [22] - both cohorts & 0.0594 & 0.0181 & 0.288 \\
\hline Brunner et al. 2000 [20] & 0.0620 & 0.0382 & 1.769 \\
\hline Heinemann et al. 1999 [30] & 0.0809 & 0.012 & 0.440 \\
\hline Danne et al. 2003 [21] & 0.0444 & 0.0072 & 0.134 \\
\hline \hline Shimoda et al. 1997 [34] & 0.0029 & - & - \\
\hline Wong et al. 2008 [35] & - & 0.0649 & - \\
\hline Lotz et al. 2008 [24] & - & - & $0.22^{*}$ \\
\hline
\end{tabular}

*Value is the sum of the hepatic and renal clearances in Lotz et al.

\subsection{Comparison to literature reported PK outcomes for identified models for each data set:}

To evaluate the model fit for each individual identified data set PK parameters $A U C, C_{\max }$ and $t_{\max }$ are compared in Table 5, where a tick ( $)$ indicates the model results within 1 SD criteria is met. In all cases, the individually identified models were able to match reported literature kinetics within $1 \mathrm{SD}$, indicating the model was able to capture all key pharmacokinetics of insulin Detemir for each data set and study, individually. The corresponding identified model fits are shown in Figure 2.

Table 5: Comparison of the model to literature reported PK outcomes for the individually identified data sets. A tick $(\checkmark)$ indicates criteria of within 1 SD is met, a cross $(X)$ is unmet.

\begin{tabular}{|c|c|c|c|c|c|c|c|c|c|c|}
\hline \multirow[t]{2}{*}{ Paper } & \multirow{2}{*}{$\begin{array}{l}\text { Dose } \\
\text { Size } \\
\text { ( U/kg) }\end{array}$} & \multicolumn{3}{|c|}{$\operatorname{AUC}\left(\mathrm{pmol} \cdot \mathrm{min} / \mathrm{L} \cdot 10^{6}\right)$} & \multicolumn{3}{|c|}{$C_{\max }\left(\mathrm{pmol} / \mathrm{L} \times 10^{3}\right)$} & \multicolumn{3}{|c|}{$t_{\max }$ (hours) } \\
\hline & & Model & Data \pm SD & & Model & Data \pm SD & & Model & Data \pm SD & \\
\hline \multirow{3}{*}{$\begin{array}{l}\text { Jhee et al. } 2004 \text { [22] } \\
\text { Caucasian cohort }\end{array}$} & 0.19 & 1.06 & $0.99 \pm 0.15$ & $\checkmark$ & 1.38 & $1.82 \pm 0.5$ & $\checkmark$ & 4.98 & $5.38 \pm 2.01$ & $\checkmark$ \\
\hline & 0.38 & 2.40 & $2.39 \pm 0.36$ & $\checkmark$ & 3.46 & $4.14 \pm 1.16$ & $\checkmark$ & 4.95 & $6.0 \pm 2.28$ & $\checkmark$ \\
\hline & 0.75 & 4.87 & $4.65 \pm 0.70$ & $\checkmark$ & 5.49 & $6.88 \pm 1.93$ & $\checkmark$ & 6.95 & $6.23 \pm 2.10$ & $\checkmark$ \\
\hline \multirow{3}{*}{$\begin{array}{l}\text { Jhee et al. } 2004 \text { [22] } \\
\text { Japanese cohort }\end{array}$} & 0.19 & 0.99 & $0.97 \pm 0.15$ & $\checkmark$ & 1.47 & $1.74 \pm 0.49$ & $\checkmark$ & 4.46 & $4.53 \pm 1.97$ & $\checkmark$ \\
\hline & 0.38 & 2.21 & $2.11 \pm 0.32$ & $\checkmark$ & 2.66 & $3.18 \pm 0.89$ & $\checkmark$ & 5.58 & $6.03 \pm 2.62$ & $\checkmark$ \\
\hline & 0.75 & 4.87 & $4.29 \pm 0.64$ & $\checkmark$ & 5.49 & $5.80 \pm 1.60$ & $\checkmark$ & 6.95 & $6.93 \pm 2.40$ & $\checkmark$ \\
\hline \multirow[t]{2}{*}{ Brunner et al. 2000 [20] } & 0.3 & 0.49 & $0.49 \pm 0.10$ & $\checkmark$ & 0.93 & $1.18 \pm 0.39$ & $\checkmark$ & 3.02 & $3.6 \pm 1.17$ & $\checkmark$ \\
\hline & 0.6 & 0.95 & $0.93 \pm 0.14$ & $\checkmark$ & 1.52 & $1.81 \pm 0.48$ & $\checkmark$ & 3.96 & $4.5 \pm 2.07$ & $\checkmark$ \\
\hline \multirow[t]{3}{*}{ Heinemann et al. 1999 [30] } & 0.15 & 0.33 & $0.34 \pm 0.04$ & $\checkmark$ & 0.55 & $0.61 \pm 0.14$ & $\checkmark$ & 3.98 & $4.45 \pm 1.4$ & $\checkmark$ \\
\hline & 0.3 & 0.63 & $0.67 \pm 0.08$ & $\checkmark$ & 0.94 & $1.05 \pm 0.28$ & $\checkmark$ & 5.01 & $5.42 \pm 1.6$ & $\checkmark$ \\
\hline & 0.6 & 1.19 & $1.23 \pm 0.21$ & $\checkmark$ & 1.85 & $2.03 \pm 0.46$ & $\checkmark$ & 5.03 & $5.37 \pm 1.2$ & $\checkmark$ \\
\hline Danne et al. 2003 [21] & 0.5 & 3.28 & $4.64 \pm 2.30$ & $\checkmark$ & 3.54 & $3.38 \pm 1.42$ & $\checkmark$ & 7.10 & $8.05 \pm 3.43$ & 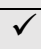 \\
\hline
\end{tabular}




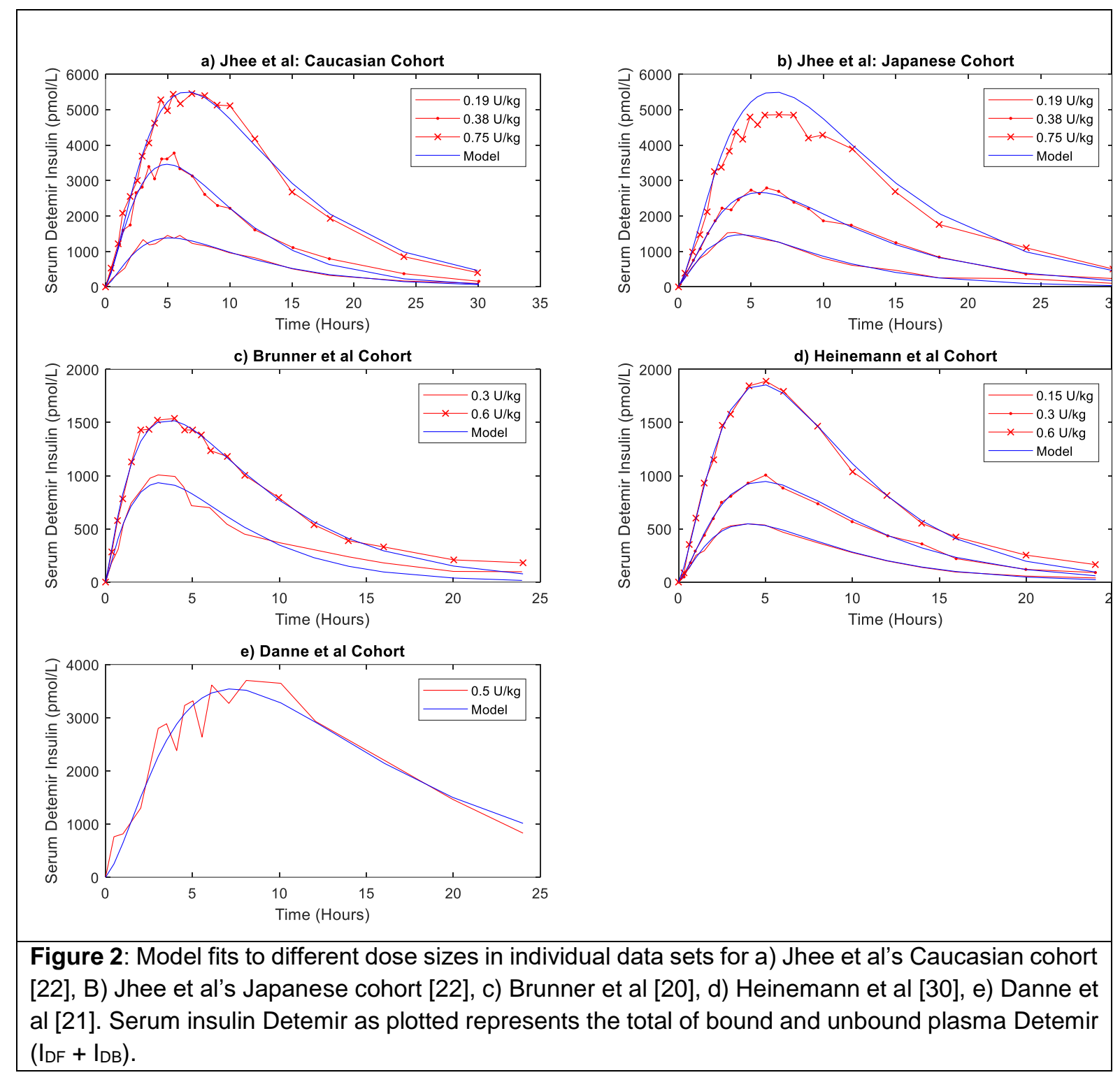

\subsection{Comparison to literature reported PK outcomes using averaged identified model values:}

The comparison of averaged parameter values from Table 4 for those data sets is shown in Figure 3 and Table 6. Due to the very large outlier values in the single data set for Brunner et al in Table 3, the averaged values for Heinemann et al were used as they have similar dose sizes.

Results for $t_{\max }$ matched the literature reported values for all data sets, and other PK outcomes matched for most data sets. However, it tended to overestimate $t_{\max }$ for lower bolus sizes and underestimate it for higher bolus sizes. This latter result suggests some model kinetics may be dependent on bolus size. 
Equally, the Jhee et al Caucasian cohort, the model underestimated AUC by more than 1 SD for the lowest Detemir bolus dose. Both of the models in the Brunner et al set underestimated AUC and were outside of $1 \mathrm{SD}$ of literature values. This latter results is probably due to the Heinemann et al parameter values used for this simulation, but equally showing reasonable robustness across studies.

Overall, there is a small degree of dose specific response to insulin Detemir, which is not captured by averaged values across a study. This inter-study variability is reflected in Table 4. Equally, those results outside the conservative 1 SD criterion were within 2 SDs, which is also evident in the qualitatively good fits in Figure 3. Hence, the large number of ticks in Table 6 (32 of 36 possible comparisons, $88 \%$ ) indicates the average parameters for each study perform well, and their broad similarity indicates reasonable robustness overall.

Table 6: Validation of the model for the averaged parameters in Table 4. A tick ( $\checkmark$ ) indicates criteria of within $1 \mathrm{SD}$ is met, a cross $(X)$ is unmet.

\begin{tabular}{|c|c|c|c|c|c|c|c|c|c|c|}
\hline \multirow[t]{2}{*}{ Paper } & \multirow{2}{*}{$\begin{array}{l}\text { Dose } \\
\text { Size } \\
(\mathbf{U} / \mathbf{k g})\end{array}$} & \multicolumn{3}{|c|}{$A U C\left(\mathrm{pmol} \cdot \mathrm{hours} / \mathrm{L} \cdot 10^{6}\right)$} & \multicolumn{3}{|c|}{$C_{\max }\left(\mathrm{pmol} / \mathrm{L} \mathrm{x10^{3 } )}(0.28)\right.$} & \multicolumn{3}{|c|}{$t_{\max }$ (hours) } \\
\hline & & Model & Data & & Model & Data & & Model & Data \pm SD & \\
\hline \multirow{3}{*}{$\begin{array}{l}\text { Jhee et al. } 2004 \text { [22] } \\
\text { Caucasian cohort }\end{array}$} & 0.19 & 1.26 & $0.99 \pm 0.17$ & $X$ & 1.70 & $1.82 \pm 0.5$ & $\checkmark$ & 4.98 & $5.38 \pm 2.01$ & $\checkmark$ \\
\hline & 0.38 & 2.51 & $2.39 \pm 0.36$ & $\checkmark$ & 3.39 & $4.14 \pm 1.16$ & $\checkmark$ & 4.95 & $6.0 \pm 2.28$ & $\checkmark$ \\
\hline & 0.75 & 5.02 & $4.65 \pm 0.70$ & $\checkmark$ & 6.78 & $6.88 \pm 1.93$ & $\checkmark$ & 4.98 & $6.23 \pm 2.10$ & $\checkmark$ \\
\hline \multirow{3}{*}{$\begin{array}{l}\text { Jhee et al. } 2004 \text { [22] } \\
\text { Japanese cohort }\end{array}$} & 0.19 & 0.96 & $0.97 \pm 0.15$ & $\checkmark$ & 1.3 & $1.74 \pm 0.49$ & $\checkmark$ & 5.45 & $4.53 \pm 1.97$ & $\checkmark$ \\
\hline & 0.38 & 1.91 & $2.11 \pm 0.32$ & $\checkmark$ & 2.59 & $3.18 \pm 0.89$ & $\checkmark$ & 5.01 & $6.03 \pm 2.62$ & $\checkmark$ \\
\hline & 0.75 & 3.80 & $4.29 \pm 0.64$ & $\checkmark$ & 5.13 & $5.80 \pm 1.60$ & $\checkmark$ & 4.98 & $6.93 \pm 2.40$ & $\checkmark$ \\
\hline \multirow[t]{2}{*}{ Brunner et al. 2000 [20] } & 0.3 & 0.72 & $0.49 \pm 0.10$ & $X$ & 1.15 & $1.18 \pm 0.39$ & $\checkmark$ & 4.50 & $3.6 \pm 1.17$ & $\checkmark$ \\
\hline & 0.6 & 1.43 & $0.93 \pm 0.14$ & $\mathrm{X}$ & 2.30 & $1.81 \pm 0.48$ & $\mathrm{X}$ & 4.55 & $4.5 \pm 2.07$ & $\checkmark$ \\
\hline \multirow[t]{3}{*}{ Heinemann et al. 1999 [30] } & 0.15 & 0.36 & $0.34 \pm 0.04$ & $\checkmark$ & 0.57 & $0.61 \pm 0.14$ & $\checkmark$ & 3.98 & $4.45 \pm 1.4$ & $\checkmark$ \\
\hline & 0.3 & 0.71 & $0.67 \pm 0.08$ & $\checkmark$ & 1.14 & $1.05 \pm 0.28$ & $\checkmark$ & 4.00 & $5.42 \pm 1.6$ & $\checkmark$ \\
\hline & 0.6 & 1.43 & $1.23 \pm 0.21$ & $\checkmark$ & 2.29 & $2.03 \pm 0.46$ & $\checkmark$ & 4.05 & $5.37 \pm 1.2$ & $\checkmark$ \\
\hline Danne et al. 2003 [21] & 0.5 & 3.29 & $4.64 \pm 2.30$ & $\checkmark$ & 3.54 & $3.38 \pm 1.42$ & $\checkmark$ & 7.10 & $8.05 \pm 3.43$ & $\checkmark$ \\
\hline
\end{tabular}




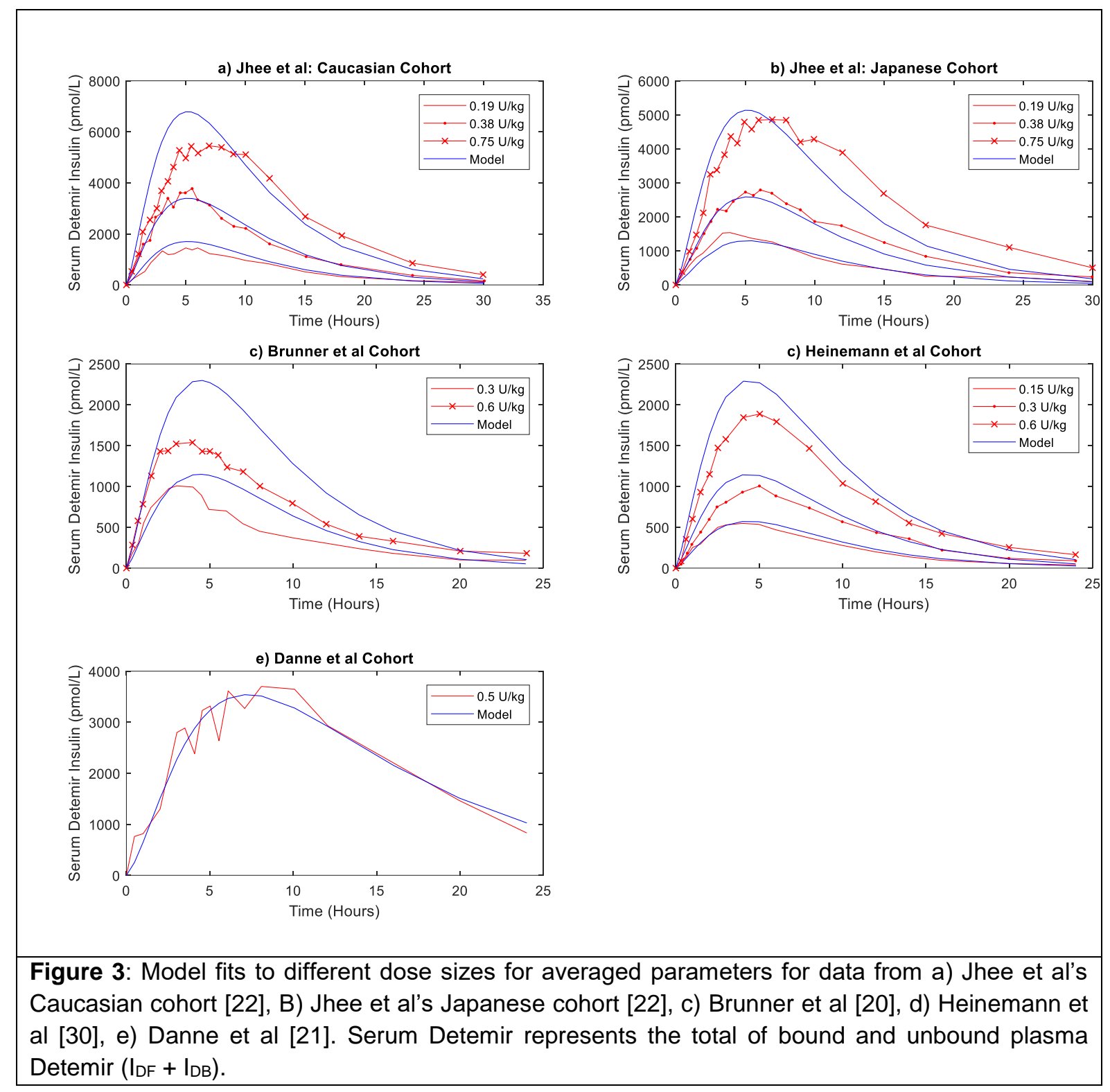

Finally, to assess average kinetic parameters, kinetic parameter values were averaged across the Heinemann et al and Jhee et al data sets and the model fits are shown in Figure 4. The inter study variability leads to the model fit underestimating the Jhee et al data and overestimating the Heinemann et al data. Again, these results indicate dose specific and/or cohort specific dynamics not fully capture although qualitative fits are acceptable. 


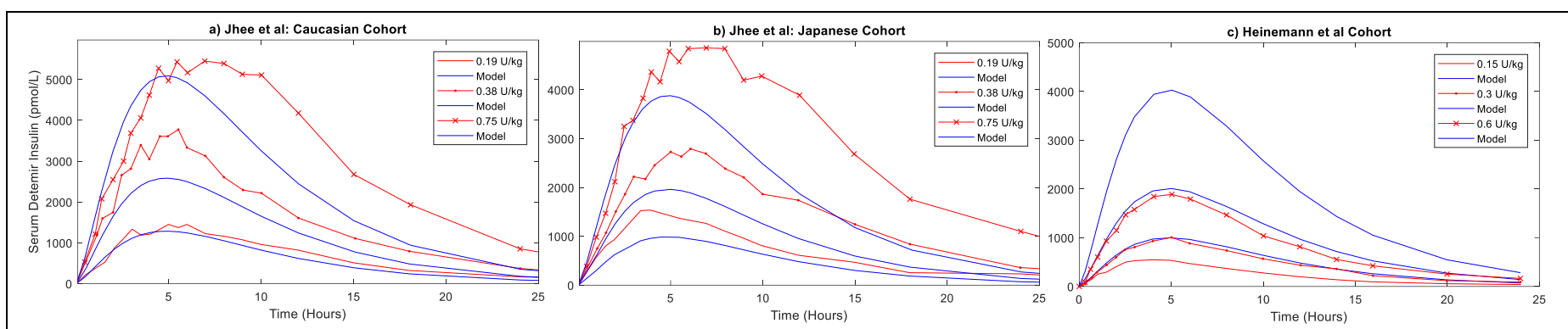

Figure 4: Model fits to different dose sizes for averaged parameters across both the Jhee and Heinemann studies for data from a) Jhee et al's Caucasian cohort [22], B) Jhee et al's Japanese cohort [22], and c) Heinemann et al [30]. Serum Detemir represents the total of bound and unbound plasma Detemir (IDF $\left.+\mathrm{IDB}_{\mathrm{DB}}\right)$ 


\subsection{Discussion:}

A model was fit to reported literature pharmacokinetics of insulin Detemir, and was able to achieve good model fits and match most reported literature pharmacokinetics to within the experimentally reported 1 SD for both healthy, and T2D patients. The model is thus able to capture all major kinetics, and can be used to model the appearance of subcutaneously administered insulin Detemir. The rate constant from the local interstitium to the blood plasma, $k_{b}$, was consistently much lower than the value for human insulin [23]. This outcome suggests there is an extra prolonging mechanism not in the model or the FFA chain slows trans-capillary diffusion requiring a different assumption and a new parameter fit.

Insulin Detemir is able to bind to albumin in its hexameric form [10], which was not included in the pharmacokinetic model as the percentage binding in hexameric form is likely much lower [10]. This dynamic may explain the lower rate of $k_{b}$, as it would slow down the insulin absorption into the blood plasma. Additionally, $k_{b}$ in Wong is modelled using a much lower value for $V_{I}$, the volume of distribution, which would affect $k_{b}$. Overall, $k_{b}$ is relatively consistent across all studies in this analysis and together $k_{a}$ and $k_{b}$ seem to acceptably control the overall appearance of Detemir insulin into plasma, where it is this overall appearance that is important to the model.

The degradation rate of insulin in the local interstitium, $k_{d i}$, was much higher than suggested by the literature [23]. This result suggests insulin Detemir is preferentially cleared from this space, or there is another loss of insulin Detemir not accounted for in the model or reported in the literature we have seen. However Wong et al's study included $k_{d i}$ acting on multiple compartments in the subcutaneous model, where here it only acts on free monomeric/dimeric Detemir at the depot. It was also derived using a lower $V_{I}$ value than in Wong et al based on newer information. Equally, the fraction of bound insulin Detemir could be different to the value of $96 \%$ considered in this study, where there are reports as high as $98-99 \%$, although literature suggests bound rates are similar between interstitial fluid and blood plasma $[10,12,26-28]$.

The identified hepatic/renal clearance constant, $n_{D L}$, is very similar in value to the literature [24]. The exception is the data from Brunner et al, where peak insulin Detemir concentrations were much lower 
at similar dose sizes in comparison to other studies, suggesting an added un-modelled loss. Overall, the loss in Brunner et al is extremely high, as well, and may reflect the particular study cohort, methodology, or assay conditions. The overall comparable $n_{D L}$ value suggests unbound insulin is able to be cleared through the liver at a similar rate to human insulin.

One limitation is the literature reported values for $C_{\max }$ often differed from the corresponding graphed study results by $13.5 \pm 7.6 \%$ (mean $\pm S D$ ). This difference may lead to inaccuracy in the comparison method since the kinetic parameters are determined by fitting to the graphical data and the model fits are compared to the reported values from the original papers. It is unclear why this contradiction exists within the original literature studies. If this extra source of variation is included, then model derived values for $C_{\max }$ are within the acceptable range for all data sets, and for the average study identified parameter values, which significantly improves the reported results.

As literature suggests the fraction of bound Detemir insulin is approximately similar in the blood plasma and interstitial fluid, this fraction (affecting $k_{1}$ and $k_{2}$ ) was held constant in all compartments. This percentage bound is approximately $96-98 \%$ at steady state $[10,12]$. The value of $96 \%$ was used as it improved model convergence, while maintaining biologically relevant numbers and results. The model could be identified for higher values of the binding constant and would give almost identical concentrations in the blood plasma accompanied by an increase in the fitted kinetic parameters.

Dea et al [12] compared human insulin to insulin Detemir in dogs to determine if the rate constants for the Detemir clearance were reduced based on the binding affinity to albumin. Dea et al found that $96.4 \%$ of Detemir would be albumin bound in interstitial fluid, and $98.3 \%$ in blood plasma. However, due to the simplicity of their two compartmental model, and minimal amount of data, the difference between the two binding values is likely to be clinically insignificant and statistically insignificant in the study report. There is also no proposed physiological explanation as to why binding percentages would be different in the plasma and interstitial fluid. Dea et al also noted the transport of insulin Detemir from the blood plasma to interstitium was higher than expected based on their binding predictions, suggesting there was more unbound insulin Detemir than predicted, which would justify a lower value. Thus, the $96 \%$ 
bound value used here is justified based on limited literature data available and the inability to assess a better value without further extended tests.

The study by Danne et al involved a cohort of Type I diabetic patients, compared to the other studies that used healthy cohorts (Table 2). The parameter values $k_{b}$ and $n_{D L}$ were both lower for the Danne et al cohort $\left(k_{b}=0.044, n_{D L}=0.134\right)$ than the values for the healthy cohorts ( $k_{b}=0.0594$ to 0.0809 , and $n_{D L}=0.288$ to 0.440 ). This difference could potentially be caused by diabetic patients being much more insulin resistant, so insulin is used more slowly and remains in blood longer, leading to higher $T_{\max }$ and $C_{\max }$ [36]. There is also the potential for kidney failure to some level in this cohort [37], so $n_{K}$ would be lower and would cause a higher $C_{\max }$. In the first case, a separate model would be required for type 1 diabetes patients, and in the second a different value for kidney clearance would have to be used in identifying the model parameters, which was not the case here.

There was a large variation in serum concentrations for the different studies even at similar bolus amounts and especially for the Jhee et al study compared to the Heinemann et al and Brunner et al studies. The most probable reason for this variability is differences in the methods used. The basic design of the three studies was similar in the fact patients were fasted beforehand, only received intravenous infusions of glucose during the test, and received a subcutaneous injection of insulin Detemir. The Jhee et al cohort had the most prolonged action and this longer action could be because they received the subcutaneous injection in the thigh, where, in contrast, the Brunner et al, and Heinemann et al cohorts both received a para-umbilical skin fold injection. The Heinemann cohort also received an intravenous infusion of human insulin throughout the study. These differences may account for differences in parameter values where greater consistency might otherwise be expected.

A different potential source of this error is body weight, where weight varied between and within study cohorts. A constant volume of distribution for insulin in plasma of $4.0 \mathrm{~L}$ was used in this analysis, based on previous work and non-reporting of weight in one study. However, as weights vary, it could explain some of the dose-dependent variability across studies, as seen for example between the Japanese and Caucasian cohorts in the Jhee et al study (Table 2). Thus, a similar sized $\mathrm{U} / \mathrm{kg}$ dose can appear very differently in different subjects and studies. This error could shift curves by $+/-10-20 \%$ at most, or equally 
this value improves the qualitative comparisons in Figures 3-4. Therefore, further studies to validate the model need to include weight and/or BMI explicitly so subject specific volumes of distribution can be employed $[38,39]$.

However, it is important to note the kinetics of insulin appearance, which is the main goal of this model are not a function of cohort. Disappearance from plasma due to action at the receptor is where cohort differences arise. Thus, the three parameters identified are all related to the specific kinetics of the drug in the depot and plasma, and, critically, not its action. There are no reported reasons in our knowledge to indicate the existence of different diffusion/transport kinetics for insulin between the interstitial fluid and plasma for these populations. Hence, it is unlikely there are significant differences between T1D and T2D cohort parameters, although it remains to be demonstrated on larger trials with specific cohorts which the results here could justify.

Further, all five studies measured the serum insulin Detemir by an enzyme-linked immunosorbent assay (ELISA) developed by NovoNordisk using monoclonal antibodies recognizing the acylation site of insulin Detemir. Thus, dosing area and method may cause this variability, while measurement method was consistent. Since there was also no weights provided for the subjects in the Heinemann and Brunner cohorts these values had to be estimated which contributes to the variability as it modifies the distribution volumes in the modeling of any pharmacokinetics and is particularly sensitive in insulin kinetics studies using this overall model framework [40-44]. In addition, assay methods should be carefully noted and kept consistent to minimise variability. Equally, the test method should be consistent across cohorts.

Finally, The 7 compartment, 8 parameter model complexity can be considered a drawback, particularly with regard to identifiability of parameters to ensure an accurate, robust model $[33,45,46]$. Analysis of the current and several prior (not shown) model structures indicated simpler models were not able to capture the pharmacokinetic profiles accurately with lesser compartments or parameters. The cause is the bound/free action of Detemir with albumin, which is what makes it unique. As a result, this action also requires including both these compartments to accurately capture its appearance profile. 
More specifically, the rate parameters for this binding are easily estimated from published ratios of free and bound Detemir. The latter clearance and diffusion between whole body plasma and interstitium is based on an existing, well-validated model of insulin kinetics. Thus, the key compartments for potential simplification are the local depot compartments, where they proved necessary to capture the peak and decay rate of the published appearance profiles. Hence, the model presented is actually the simpler of some others examined that is able to capture this unique action and its resulting impact on appearance kinetics.

As a result of the limitations noted, the model developed in this study should be validated against clinical data from an independent cohort, particularly one focusing on T2D for this application. Better, more extensive controlled experiments across the cohorts in the studies used here, particularly target cohorts with T1D and T2D, would enable better determination of the robustness of the model, where the variability limits the ability of this analysis to provide more than a proof of concept validation. However, these results justify those clinical trials on specialised cohorts. 


\subsection{Conclusions:}

The model developed in this study provides acceptable model fits, is robust across cohorts to within measurement error, although limited by variations in study methods and assays. All observed dynamics appear to be captured, although some dose specific dynamics may exist, and potentially cohort specific dynamics for individuals with type 1 diabetes. The overall modeling approach may also be generalisable to other drugs utilising a similar albumin binding mechanism for transport and delayed action. The overall results show the model is a physiologically accurate representation of insulin Detemir pharmacokinetics in the human body, and can thus be used in model-based analyses of glycemia and glycemic control, although further extended tests with greater consistency in methods and assays are suggested to validate the initial proof of concept model and analysis presented here.

\subsection{Acknowledgements:}

Funding: this research was partly funded by the New Zealand National Science Challenge Science for Technological Innovation (SfTI), sourced from the NZ Tertiary Education Commission (TEC), grant number CRS-S3-2016.

Ethics Approval: The data used in this study was sourced from published literature and no new clinical data was used. There is thus no need for ethics approval.

Competing Interests: The authors declare no competing or financial interest in this work. 


\subsection{References}

1. Shaw, J.E., R.A. Sicree, and P.Z. Zimmet, Global estimates of the prevalence of diabetes for 2010 and 2030. Diabetes Res Clin Pract, 2010. 87(1): p. 4-14.

2. Whiting, D.R., L. Guariguata, C. Weil, and J. Shaw, IDF diabetes atlas: global estimates of the prevalence of diabetes for 2011 and 2030. Diabetes Res Clin Pract, 2011. 94(3): p. 311-21.

3. Wild, S., G. Roglic, A. Green, R. Sicree, and H. King, Global prevalence of diabetes: estimates for the year 2000 and projections for 2030. Diabetes Care, 2004. 27(5): p. 1047-53.

4. Zhang, P., X. Zhang, J. Brown, D. Vistisen, R. Sicree, J. Shaw, and G. Nichols, Global healthcare expenditure on diabetes for 2010 and 2030. Diabetes Res Clin Pract, 2010. 87(3): p. 293-301.

5. $\quad$ Chatterjee, S., K. Khunti, and M.J. Davies Type 2 Diabetes. The Lancelet, 2017. 389(10085): p. 12.

6. Wallace, J.P., J.L. Wallace, and M.S. McFarland, Comparing dosing of basal insulin analogues detemir and glargine: is it really unit-per-unit and dose-per-dose? Ann Pharmacother, 2014. 48(3): p. 361-8.

7. Batais, M.A. and P. Schantter, Prevalence of unwillingness to use insulin therapy and its associated attitudes amongst patients with Type 2 diabetes in Saudi Arabia. Prim Care Diabetes, 2016. 10(6): p. 415-424.

8. Philis-Tsimikas, A., Initiating basal insulin therapy in type 2 diabetes: practical steps to optimize glycemic control. Am J Med, 2013. 126(9 Suppl 1): p. S21-7.

9. Edelman, S. and J. Pettus, Challenges associated with insulin therapy in type 2 diabetes mellitus. Am J Med, 2014. 127(10 Suppl): p. S11-6.

10. Havelund, S., A. Plum, U. Ribel, I. Jonassen, A. Volund, J. Markussen, and P. Kurtzhals, The mechanism of protraction of insulin detemir, a long-acting, acylated analog of human insulin. Pharm Res, 2004. 21(8): p. 1498-504.

11. Banks, W.A., J.E. Morley, J.L. Lynch, K.M. Lynch, and A.D. Mooradian, Insulin detemir is not transported across the blood-brain barrier. Peptides, 2010. 31(12): p. 2284-8.

12. Dea, M.K., M. Hamilton-Wessler, M. Ader, D. Moore, L. Schaffer, M. Loftager, A. Volund, and R.N. Bergman, Albumin binding of acylated insulin (NN304) does not deter action to stimulate glucose uptake. Diabetes, 2002. 51(3): p. 762-9.

13. Thabit, H., S. Hartnell, J.M. Allen, A. Lake, M.E. Wilinska, Y. Ruan, M.L. Evans, A.P. Coll, and R. Hovorka, Closed-loop insulin delivery in inpatients with type 2 diabetes: a randomised, parallelgroup trial. Lancet Diabetes Endocrinol, 2017. 5(2): p. 117-124.

14. Rowley, W.R., C. Bezold, Y. Arikan, E. Byrne, and S. Krohe, Diabetes 2030: Insights from Yesterday, Today, and Future Trends. Popul Health Manag, 2017. 20(1): p. 6-12.

15. Bally, L., H. Thabit, S. Hartnell, E. Andereggen, Y. Ruan, M.E. Wilinska, M.L. Evans, M.M. Wertli, A.P. Coll, C. Stettler, and R. Hovorka, Closed-Loop Insulin Delivery for Glycemic Control in Noncritical Care. N Engl J Med, 2018. 379(6): p. 547-556.

16. Wong, J., J.G. Chase, C.E. Hann, T.F. Lotz, J. Lin, A. Le Compte, and G.M. Shaw, In Silico Simulation of Long-Term Type 1 Diabetes Glycemic Control Treatment Outcomes. Journal of Diabetes Science and Technology, 2008. 2(3): p. 425-435.

17. Wong, J., J.G. Chase, C.E. Hann, G.M. Shaw, T.F. Lotz, J. Lin, and A.J. Le Compte, A Subcutaneous Insulin Pharmacokinetic Model for Computer Simulation in a Diabetes Decision Support Role: Model Structure and Parameter Identification. Journal of Diabetes Science and Technology, 2008. 2(4): p. 658-671.

18. Wong, J., J.G. Chase, C.E. Hann, G.M. Shaw, T.F. Lotz, J. Lin, and A.J. Le Compte, A Subcutaneous Insulin Pharmacokinetic Model for Computer Simulation in a Diabetes Decision Support Role: Validation and Simulation. Journal of Diabetes Science and Technology, 2008. 2(4): p. 672-680. 
19. Tarin, C., E. Teufel, J. Pico, J. Bondia, and H.J. Pfleiderer, Comprehensive pharmacokinetic model of insulin Glargine and other insulin formulations. IEEE transactions on bio-medical engineering, 2005. 52(12): p. 1994-2005.

20. Brunner, G.A., G. Sendhofer, A. Wutte, M. Ellmerer, B. Sogaard, A. Siebenhofer, S. Hirschberger, G.J. Krejs, and T.R. Pieber, Pharmacokinetic and pharmacodynamic properties of long-acting insulin analogue NN304 in comparison to NPH insulin in humans. Exp Clin Endocrinol Diabetes, 2000. 108(2): p. 100-5.

21. Danne, T., K. Lupke, K. Walte, W. Von Schuetz, and M.A. Gall, Insulin detemir is characterized by a consistent pharmacokinetic profile across age-groups in children, adolescents, and adults with type 1 diabetes. Diabetes Care, 2003. 26(11): p. 3087-92.

22. Jhee, S.S., W.H. Lyness, P.B. Rojas, M.T. Leibowitz, V. Zarotsky, and L.V. Jacobsen, Similarity of insulin detemir pharmacokinetics, safety, and tolerability profiles in healthy caucasian and Japanese american subjects. J Clin Pharmacol, 2004. 44(3): p. 258-64.

23. Wong, J., Model-Based Therapeutics for Type 1 Diabetes Mellitus. 2008, University of Canterbury: Christchurch, New Zealand. p. 259.

24. Lotz, T.F., J.G. Chase, K.A. McAuley, G.M. Shaw, X.W. Wong, J. Lin, A. Lecompte, C.E. Hann, and J.I. Mann, Monte Carlo analysis of a new model-based method for insulin sensitivity testing. Comput Methods Programs Biomed, 2008. 89(3): p. 215-25.

25. Li, J. and Y. Kuang, Systemically modeling the dynamics of plasma insulin in subcutaneous injection of insulin analogues for type 1 diabetes. Math Biosci Eng, 2009. 6(1): p. 41-58.

26. Kurtzhals, P., S. Havelund, I. Jonassen, B. Kiehr, U.D. Larsen, U. Ribel, and J. Markussen, Albumin binding of insulins acylated with fatty acids: characterization of the ligand-protein interaction and correlation between binding affinity and timing of the insulin effect in vivo. Biochem J, 1995. 312 ( Pt 3): p. 725-31.

27. Kurtzhals, P., S. Havelund, I. Jonassen, and J. Markussen, Effect of fatty acids and selected drugs on the albumin binding of a long-acting, acylated insulin analogue. J Pharm Sci, 1997. 86(12): p. 1365-8.

28. Soran, H. and N. Younis, Insulin detemir: a new basal insulin analogue. Diabetes Obes Metab, 2006. 8(1): p. 26-30.

29. Docherty, P.D., J.G. Chase, T.F. Lotz, and T. Desaive, A graphical method for practical and informative identifiability analyses of physiological models: a case study of insulin kinetics and sensitivity. Biomed Eng Online, 2011. 10: p. 39.

30. Heinemann, L., K. Sinha, C. Weyer, M. Loftager, S. Hirschberger, and T. Heise, Time-action profile of the soluble, fatty acid acylated, long-acting insulin analogue NN304. Diabet Med, 1999. 16(4): p. 332-8.

31. Morrow, L., M. Hompesch, H. Guthrie, D. Chang, and D.J. Chatterjee, Co-administration of liraglutide with insulin detemir demonstrates additive pharmacodynamic effects with no pharmacokinetic interaction. Diabetes Obes Metab, 2011. 13(1): p. 75-80.

32. Knopp, J.L., L. Holder-Pearson, and J.G. Chase, Insulin Units and Conversion Factors: A Story of Truth, Boots, and Faster Half-Truths. J Diabetes Sci Technol, 2018: p. 1932296818805074.

33. Docherty, P.D., J.G. Chase, T.F. Lotz, and T. Desaive, A graphical method for practical and informative identifiability analyses of physiological models: A case study of insulin kinetics and sensitivity. Biomedical Engineering Online, 2011. 10(1): p. 1-20.

34. Shimoda, S., K. Nishida, M. Sakakida, Y. Konno, K. Ichinose, M. Uehara, T. Nowak, and M. Shichiri, Closed-loop subcutaneous insulin infusion algorithm with a short-acting insulin analog for long-term clinical application of a wearable artificial endocrine pancreas. Front Med Biol Eng, 1997. 8(3): p. 197-211.

35. Wong, J., J.G. Chase, C.E. Hann, G.M. Shaw, T.F. Lotz, J. Lin, and A.J. Compte, A subcutaneous insulin pharmacokinetic model for computer simulation in a diabetes decision support role: validation and simulation. J Diabetes Sci Technol, 2008. 2(4): p. 672-80. 
36. Priya, G. and S. Kalra, A Review of Insulin Resistance in Type 1 Diabetes: Is There a Place for Adjunctive Metformin? Diabetes Ther, 2018. 9(1): p. 349-361.

37. Coresh, J., B.C. Astor, T. Greene, G. Eknoyan, and A.S. Levey, Prevalence of chronic kidney disease and decreased kidney function in the adult US population: Third National Health and Nutrition Examination Survey. Am J Kidney Dis, 2003. 41(1): p. 1-12.

38. Turnheim, K. and W.K. Waldhausl, Essentials of insulin pharmacokinetics. Wien Klin Wochenschr, 1988. 100(3): p. 65-72.

39. Despopoulos, A. and S. Silbernagl, Color atlas of physiology. 5th ed. Thieme flexibook. 2003, Stuttgart New York: G. Thieme

Thieme Medical Publishers. $x, 448 \mathrm{~s}$.

40. Docherty, P.D., J.G. Chase, T. Lotz, C.E. Hann, G.M. Shaw, J.E. Berkeley, J. Mann, and K. McAuley, DISTq: an iterative analysis of glucose data for low-cost, real-time and accurate estimation of insulin sensitivity. The Open Medical Informatics Journal, 2009. 3: p. 65.

41. Docherty, P.D., J.G. Chase, T.F. Lotz, C.E. Hann, G.M. Shaw, J.E. Berkeley, L. TeMorenga, J.I. Mann, and K. McAuley, Independent cohort cross-validation of the real-time DISTq estimation of insulin sensitivity. Computer Methods and Programs in Biomedicine, 2011. 102(2): p. 94104.

42. Docherty, P.D., J.G. Chase, L. Morenga, T.F. Lotz, J. Berkeley, G. Shaw, K. McAuley, and J. Mann, A spectrum of dynamic insulin sensitivity test protocols. Journal of Diabetes Science and Technology, 2011. 5(6): p. 1499.

43. Lotz, T., High Resolution Clinical Model-Based Assessment of Insulin Sensitivity, in Mechanical Engineering. 2007, University of Canterbury: Christchurch. p. 231.

44. Lotz, T.F., J.G. Chase, K.A. McAuley, G.M. Shaw, P.D. Docherty, J.E. Berkeley, S.M. Williams, C.E. Hann, and J.I. Mann, Design and clinical pilot testing of the model-based Dynamic Insulin Sensitivity and Secretion Test (DISST). Journal of Diabetes Science and Technology, 2010. 4(6): p. 1408-1423.

45. Chase, J.G., J.C. Preiser, J.L. Dickson, A. Pironet, Y.S. Chiew, C.G. Pretty, G.M. Shaw, B. Benyo, K. Moeller, S. Safaei, M. Tawhai, P. Hunter, and T. Desaive, Next-generation, personalised, model-based critical care medicine: a state-of-the art review of in silico virtual patient models, methods, and cohorts, and how to validation them. Biomed Eng Online, 2018. 17(1): p. 24.

46. Docherty, P.D., J.G. Chase, and T. David, Characterisation of the iterative integral parameter identification method. Medical and Biological Engineering and Computing, 2012: p. 1-8. 